\title{
An Invariant Shape Representation Using the Anisotropic Helmholtz Equation
}

\author{
A.A. Joshi ${ }^{1}$, S. Ashrafulla ${ }^{1}$, D.W. Shattuck ${ }^{2}$, H. Damasio ${ }^{3}$, and R.M. Leahy ${ }^{1, \star}$ \\ 1 Signal \& Image Processing Institute, Univ. of Southern California, Los Angeles, CA \\ 2 Laboratory of Neuro Imaging, Univ. of California, Los Angeles, CA \\ 3 Brain and Creativity Institute, Univ. of Southern California, Los Angeles, CA
}

\begin{abstract}
Analyzing geometry of sulcal curves on the human cortical surface requires a shape representation invariant to Euclidean motion. We present a novel shape representation that characterizes the shape of a curve in terms of a coordinate system based on the eigensystem of the anisotropic Helmholtz equation. This representation has many desirable properties: stability, uniqueness and invariance to scaling and isometric transformation. Under this representation, we can find a point-wise shape distance between curves as well as a bijective smooth point-to-point correspondence. When the curves are sampled irregularly, we also present a fast and accurate computational method for solving the eigensystem using a finite element formulation. This shape representation is used to find symmetries between corresponding sulcal shapes between cortical hemispheres. For this purpose, we automatically generate 26 sulcal curves for 24 subject brains and then compute their invariant shape representation. Left-right sulcal shape symmetry as measured by the shape representation's metric demonstrates the utility of the presented invariant representation for shape analysis of the cortical folding pattern.
\end{abstract}

\section{Introduction}

The human cerebral cortex is a highly convoluted sheet with rich and detailed folding patterns. Sulci are fissures in the cortical surface which are used frequently as anatomical landmarks. The geometry of these cortical landmarks is used for registration as well as the study of disease progression [10, aging [12] and brain asymmetry [1]. However, these approaches do not use the shapes of sulci but instead features such as length, depth and 3D location.

Quantification, matching, and classification of the shape of curves is a challenging problem with a long history. Spectral graphs [3] use graph theory to attempt to match two curves. In addition, geometric features such as areas of enclosed regions [21] have been used for curve representation. Recent methods use the distributions of distances from all points on a curve to a reference point; the most popular is the shape context [9].

\footnotetext{
* This work was supported by grants NIH-NIBIB P41 EB015922 / P41 RR 013642 and NIH-NINDS R01 NS074980.
} 
PDE based models such as elastic matching [19] LDDMM [5], conformal representation [16], bimorphisms [20] use locally smooth deformation models for curve registration. In general, these methods find transform parameters and then calculate a distance on the final fit [2. However, it is unclear whether they capture both the local and the global features of a curve.

In order to address these issues in the context of sulcal pattern analysis, we present a novel model for shape analysis of 1D curves based on an extension of the Global Point Signature (GPS) [1314]. The GPS representation for 2D surfaces uses the eigensystem of the Laplace-Beltrami operator. We first review our method for labeling the cortical surface automatically and for generating sulci on a population of cortical surfaces. We next present a novel invariant 1D curve representation based on the extension of GPS to $1 \mathrm{D}$ curves. For consistency of terminology with the shape analysis literature, we refer to our novel representation as the GPS representation of curves. 1D curves are represented using the eigensystem of an anisotropic Helmholtz equation where curvature is used as the anisotropy term. This representation also defines a metric in the embedded space of the representation - admitting a local measure of shape distance for curve matching. Finally, we present the results of applying our methodology towards analysis of sulcal shape symmetry between left and right brain hemispheres.

\section{Sulci Generation}

We briefly review our method for automatic generation of sulci on a subject's cortical surface, described in more detail in [7]. We assume as input a triangulated mesh approximating the cortical surface. We use BrainSuite [17] to extract the cortical surface meshes from T1-weighted MRI volumes for an atlas and for the subject. We then identify sulcal landmarks on the cortex automatically. We compute a one-to-one correspondence between the atlas surface and the subject surface in two stages: (i) the surface of each cortical hemisphere is parameterized to a unit square, and (ii) a vector field is found within this parameterization that aligns curvature of the atlas surface to curvature of the subject surface. We parameterize the cortical surfaces by modeling them as an elastic sheet and then solving the associated linear elastic equilibrium equation using finite elements. We constrain the corpus callosum to lie uniformly on the boundary of the unit square. The elastic energy minimization yields flat maps of each cortical hemisphere to a plane (Fig. 1). Multiresolution representations of curvature for the subject and atlas are calculated and then aligned by minimizing a cost function with elastic energy as a regularizer. This step reparameterizes the cortical hemisphere surfaces, establishing a one-to-one point correspondence between subject and atlas surfaces.

For this study we registered $N=24$ T1-weighted MRI volumes. For the atlas, a set of 26 sulcal curves per hemisphere were traced interactively in BrainSuite [17] using the protocol described in [1]. Using the point correspondence 
established above, these sulci are transferred to the subject surface. The locations of the estimates are subsequently refined using geodesic curvature flow on the cortical surface as described in [7. The refinement uses a level set based formulation of flow on non-flat surfaces with the sulci as the zero level sets. The resulting $\mathrm{PDE}$ is discretized on a triangulated mesh using finite elements. After this refinement, we have 26 sulci on each of $N=24$ cortical surfaces. We note that other methods for sulcal set generation [18] can also be used.

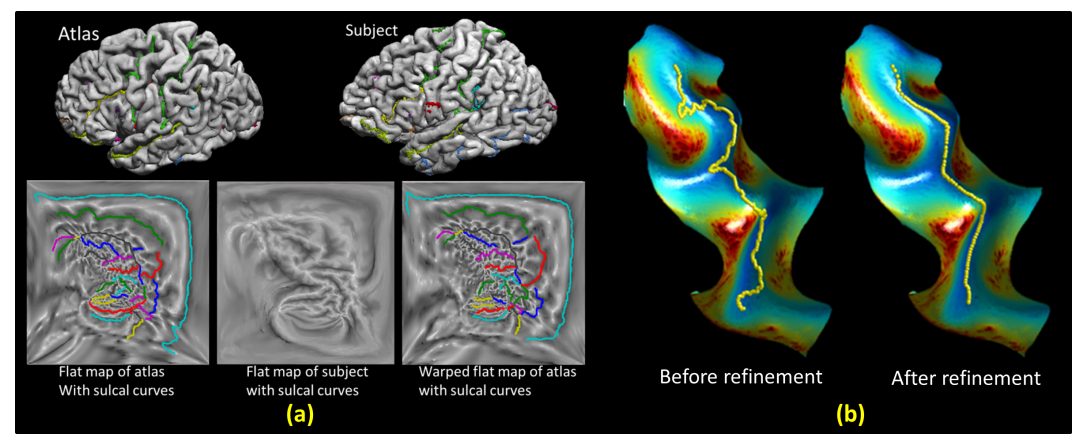

Fig. 1. (a) Automatic atlas to subject registration and parameterization of cortical surfaces and sulcal curves; and (b) geodesic curvature flow refinement of sulcal curves

\section{Shape Representation Using GPS Representation}

In this section we introduce a coordinate system to represent a $1 \mathrm{D}$ curve. Spectral theory provides the basis to study the eigenspectrum of the sulcal curves. Motivated by spectral theory and corresponding work on 2D surfaces [14]13], we model the 1D curves as inhomogeneous vibrating strings. Their harmonic behavior is governed by the $1 \mathrm{D}$ Helmholtz equation. To characterize the shape of the curve $C$, we use its curvature $\kappa(s)$ to introduce anisotropy into the governing equation:

$$
\left\{\begin{array}{ll}
\frac{\partial}{\partial s} \kappa(s) \frac{\partial}{\partial s} \Phi_{i}(s) & =\lambda_{i} \Phi_{i}(s) \\
\left.\Phi_{i}(s)\right|_{\partial C} & =0
\end{array}, \forall s \in C\right.
$$

where $\partial C$ is the set of the endpoints of the curve $C$. Denote the eigenfunctions of this equation by $\Phi_{i}$ with eigenvalues $\lambda_{i}$ ordered by magnitude. We define the embedding manifold in the spectral domain by the map:

$$
G P S(p)=\left(\frac{1}{\sqrt{\lambda_{1}}} \Phi_{1}(p), \frac{1}{\sqrt{\lambda_{2}}} \Phi_{2}(p), \frac{1}{\sqrt{\lambda_{3}}} \Phi_{3}(p), \ldots\right) \text {. }
$$

Each point of the curve is embedded into an infinite dimensional space. We cannot use the 1D Laplacian directly for this purpose because 1D shapes always have a trivial intrinsic geometry. However, due to the fundamental theorem of curves (two unit-speed plane curves that have the same curvature and torsion differ only by a rigid transformation), curvature and torsion define the curve uniquely up to a rigid transformation. Furthermore, the curve can be recovered 


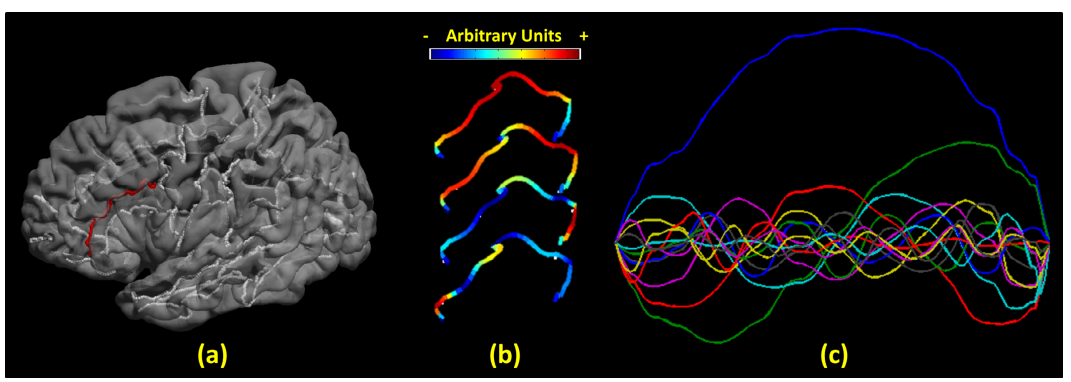

Fig. 2. (a) Inferior frontal sulcus highlighted in red; (b) first four color coded GPS coordinates; (c) GPS representation of a sulcus plotted from end to end

from the embedding by first recovering the curvature and torsion, and then using the Frenet-Serre formulas [4]. For curves in 3D space, this requires curvature and torsion. The embedding defined above is based on curvature alone because the sulcal curves analyzed in this paper had negligible torsion. The following properties also apply to this representation:

1. GPS coordinates are isometry invariant as they depend only on derivatives and curvature, which are ony dependent on shape.

2. Scaling a $1 \mathrm{D}$ curve manifold equally scales curvature. Therefore, normalizing the eigenvalue produces scale invariance (as well as position invariance, as mentioned earlier).

3. Shape changes result in continuous changes in a curve's spectrum. Consequently the representation presented here is robust.

4. In the embedding space, the inner product is given by the Green's function due to the identity: $G\left(x_{1}, x_{2}\right)=\sum_{i} \frac{\Phi_{i}\left(x_{1}\right) \Phi_{i}\left(x_{2}\right)}{\lambda_{i}}$. Thus the GPS representation encodes local and global shape information. Additionally, the metric is Euclidean.

An example of this representation for a sulcal curve is shown in Fig. 2

\section{Discretization Using Finite Element Method}

Sulcal curves are often sampled non-uniformly, so we use a finite element method to discretize the eigenvalue problem in Eq. 1. Let $\Phi(s)=\sum_{i} \phi_{i} e_{i}(s)$ be an eigenfunction and $\eta(s)=\sum_{i} \eta_{i} e_{i}(s)$ be a 'test function' represented as weighted sums of linear elements. The eigenvalue problem from Eq. 1 is:

$$
\begin{aligned}
\left(\frac{\partial}{\partial s} \kappa(s) \frac{\partial}{\partial s}\right) \Phi & =\lambda \Phi \\
\Longrightarrow \int\left(\frac{\partial}{\partial s} \kappa(s) \frac{\partial}{\partial s} \Phi(s)\right) \eta(s) d s & =\lambda \int \Phi(s) \eta(s) d s \\
\Longrightarrow \int \kappa(s) \frac{\partial}{\partial s} \Phi(s) \frac{\partial}{\partial s} \eta(s) d s & =\lambda \int \Phi(s) \eta(s) d s
\end{aligned}
$$


where the latter follows using integration by parts. Substituting the finite element model we get:

$$
\begin{aligned}
\sum_{i} \sum_{j} \phi_{i} \eta_{j} \kappa_{i j} \int \frac{\partial}{\partial s} e_{i}(s) \frac{\partial}{\partial s} e_{j}(s) d s & =\lambda \sum_{i} \sum_{j} \phi_{i} \eta_{j} \int e_{i}(s) e_{j}(s) d s \\
\kappa S \phi & =\lambda M \phi
\end{aligned}
$$

where $\kappa_{i j}$ represents $\left(\kappa_{i}+\kappa_{j}\right) / 2$ : the average of curvatures calculated at points $i$ and $j$.

For the $1 \mathrm{D}$ case with linear elements, the element-wise mass matrix is given by $M_{e l}=\left[\begin{array}{l}\left(\kappa_{i j} d_{i j}\right) / 3\left(\kappa_{i j} d_{i j}\right) / 6 \\ \left(\kappa_{i j} d_{i j}\right) / 6\left(\kappa_{i j} d_{i j}\right) / 3\end{array}\right]$ for element el corresponding to the edge between nodes $i$ and $j$. Similarly, the element-wise stiffness matrix is given by $S_{e l}=$ $\left[\begin{array}{cc}1 / d_{i j} & -1 / d_{i j} \\ -1 / d_{i j} & 1 / d_{i j}\end{array}\right]$ using linear finite elements [15].

The matrix equation in Eq. 2 is a generalized sparse eigenvalue problem that can be solved using standard methods, such as the QZ method that is a part of the Matlab function eigs. The point-wise curvature of the curve $\kappa_{i}$ is computed using the Frenet frame [4].

\section{$5 \quad$ Shape Matching}

In brain image analysis, a matching technique is required to analyze sulcal variation across a population. In this section, we describe a method for finding such a matching using GPS coordinates. Later, we match left vs right hemispherical sulci to investigate asymmetry between hemispheres.

Let $G P S_{1}$ and $G P S_{2}$ denote the GPS coordinates for the two sulcal sets. Our goal is to find a reparameterization function $\psi$ such that the matching energy $E(\psi)$ is minimized.

$$
E(\psi)=\int \|\left(G P S_{1}(s)-G P S_{2}(s+\psi(s)) \|^{2} d s\right.
$$

where $\psi$ is represented in terms of b-spline basis functions [15. Minimization of the cost function results in a 1-1 point correspondence between the two curves (Fig [3). Once the optimal $\psi$ is found, the local shape difference at point $s$ is given by $\|\left(G P S_{1}(s)-G P S_{2}(s+\psi(s)) \|\right.$.

For the purpose of mapping symmetry, we compute (a) the point-wise GPS distance between corresponding sulci from one hemisphere to the other, for all subjects; (b) the point-wise GPS distance between corresponding sulci for the same hemisphere in two different subjects. We define a measure of symmetry Symm $=-\log \left(\frac{\operatorname{mean}(a)}{\operatorname{mean}(b)}\right)$. The measure Symm ranges from 0 to $\infty$. We then use 


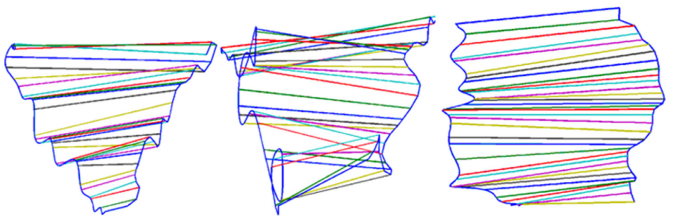

Fig. 3. Three representative sulci from left and right hemispheres and the point correspondence between them

a non-parametric Mann-Whitney-Wilcoxon test between statistics (a) and (b) at $\alpha=0.05$, correcting for multiple comparisons, with the false discovery rate (FDR).

\section{Results}

We performed symmetry detection on data from 24 subjects, divided into two cohorts of 12 subjects [11. The first cohort was scanned at the Dornsife Cognitive Neuroscience Imaging Center using a $3 \mathrm{~T}$ Siemens MAGNETOM Trio scanner. The second cohort was scanned at the University of Iowa using thin-cut MR coronal images obtained in a General Electric Signa Scanner operating at 1.5 Tesla. We applied the BrainSuite surface extraction sequence followed by sulcal set generation as outlined in Sec. 2. This produced $24 \times 2$ cortical surface hemisphere representations with 26 sulci each. The sulci were denoised by fitting a 12th order polynomial with the degree of the polynomial was selected using L-curve analysis and selecting the maximum degree necessary for all curves. Next, the GPS coordinate representation was formed as described in Sec. 3 and Sec. 4. The symmetry between the sulci was then estimated using the method in Sec 5. The results of the symmetry mapping are shown in Fig. 4. It is interesting to note that the post- and pre-central sulci, together with the posterior segment of the superior temporal, the transverse temporal, the middle temporal and the inferior occipital sulci in the dorso-lateral view, show the maximal amount of left/right asymmetry; on the mesial view the collateral, the supraorbital, the occipito-parietal and long stretches of the cingulate sulci are also extremely asymmetric. It is not surprising to see the cingulate sulcus (visible in the depth of the mesial view) show a great extent of relative symmetry. Reports of brain asymmetry usually focus on the Sylvian fissure but our data suggest that other asymmetries may be worth investigating to determine if they are indeed comparable to the well confirmed Sylvian fissure asymmetry.

It would be interesting to apply other shape representation approaches for the problem of finding sulcal shape symmetry. In this work, we tried a simple affine curve matching approach [6] that finds an optimal affine transform to minimize distance between curves but but we did not find significant asymmetries with this affine approach. 


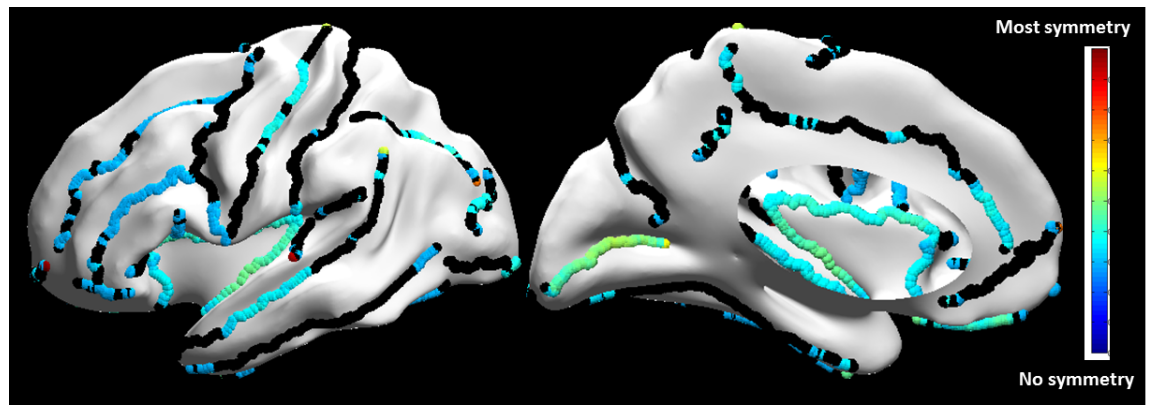

Fig. 4. Shape symmetry measure of the sulci plotted on a smooth representation of an individual cortical surface. The black regions on the curves indicate that a significant symmetry was not found for those points.

\section{Discussion and Conclusion}

We have presented a novel invariant shape representation using the eigensystem of the anisotropic Helmholtz equation. This representation also has an interesting physical interpretation in terms of vibrating strings. Because our representation depends only on shape and not on the Euclidean embedding of the shape, it is invariant to Euclidean transformations. As opposed to surface-based measures 85] in which sulcal shape differences are confounded by the shape of the cortical sheet surrounding the sulcal fold, we model sulci as curves as opposed to folds on surfaces. The invariant representation therefore provides information that is complementary to surface-based shape analysis. The properties listed Sec 3 make the presented GPS representation for curves an attractive alternative over the existing methods [2016 919], although a thorough comparison is still required.

One potential drawback of our method is that errors in automatically generated sulci can lead to inaccurate input when generating the GPS representation. We are in the process of validating the sulcal generation method in a more extensive manner on a larger data-set; initial validation is promising. It is important to note that, if required, the BrainSuite software allows for semi-automatic interactive corrections of the sulci to reduce inaccuracies.

This model has a variety of potential applications in computer vision as well as brain image analysis. Many of the existing methods for brain morphometry focus on point-wise features such as 3D location, curvature, thickness, deformation, and image intensity. Conversely, the framework we have presented directly captures the geometric shape of the folding pattern. As a result, we can study the cortical folding pattern quantitatively for a variety of neuro-developmental conditions (e.g. autism) and other neurological conditions characterized by changes in sulcal patterns. 


\section{References}

1. Blanton, R., Levitt, J., Thompson, P., Narr, K., Capetillo-Cunliffe, L., Nobel, A., Singerman, J., McCracken, J., Toga, A.: Mapping cortical asymmetry and complexity patterns in normal children. Psychiatry Research: Neuroimaging 107(1), 29-43 (2001)

2. Buchin, K., Buchin, M., Wang, Y.: Exact algorithms for partial curve matching via the Fréchet distance. In: Proc. of the 20th Ann. ACM-SIAM Symp. on Discrete Algorithms, pp. 645-654 (2009)

3. Carcassoni, M., Hancock, E.: Spectral correspondence for point pattern matching. Pattern Recognition 36(1), 193-204 (2003)

4. Do Carmo, M.: Differential Geometry of Curves and Surfaces. Prentice-Hall (1976)

5. Glaunès, J., Qiu, A., Miller, M., Younes, L.: Large deformation diffeomorphic metric curve mapping. International Journal of Computer Vision 80, 317-336 (2008)

6. Gope, C., Kehtarnavaz, N., Hillman, G., Würsig, B.: An affine invariant curve matching method for photo-identification of marine mammals. Pattern Recognition 38(1), 125-132 (2005)

7. Joshi, A.A., Shattuck, D.W., Damasio, H., Leahy, R.M.: Geodesic curvature flow on surfaces for automatic sulcal delineation. In: Proc. ISBI (in press, 2012)

8. Lai, R., Shi, Y., Sicotte, N., Toga, A.: Automated corpus callosum extraction via laplace-beltrami nodal parcellation and intrinsic geodesic curvature flows on surfaces. UCLA Computational and Applied Mathematics Reports

9. Mori, G., Belongie, S., Malik, J.: Efficient Shape Matching Using Shape Contexts. IEEE Trans. on PAMI 27(11), 1832-1837 (2005)

10. Narr, K., Thompson, P., Sharma, T., Moussai, J., Zoumalan, C., Rayman, J., Toga, A.: Three-dimensional mapping of gyral shape and cortical surface asymmetries in schizophrenia: gender effects. Am. J. Psychiatry 158(2), 244-255 (2001)

11. Pantazis, D., Joshi, A., Jiang, J., Shattuck, D., Bernstein, L., Damasio, H., Leahy, R.: Comparison of landmark-based and automatic methods for cortical surface registration. Neuroimage 49(3), 2479-2493 (2010)

12. Rettmann, M.E., Prince, J.L., Resnick, S.M.: Analysis of sulcal shape changes associated with aging. In: Proc. Human Brain Mapping, NY, June 18-22 (2003)

13. Reuter, M.: Hierarchical Shape Segmentation and Registration via Topological Features of Laplace-Beltrami Eigenfunctions. IJCV 89(2), 287-308 (2010)

14. Rustamov, R.: Laplace-beltrami eigenfunctions for deformation invariant shape representation. In: Proc. of the 5th Euro. Symp. on Geom. Proc., pp. 225-233 (2007)

15. Sadiku, M.: Numerical techniques in electromagnetics. CRC (2000)

16. Sharon, E., Mumford, D.: 2D-shape analysis using conformal mapping. International Journal of Computer Vision 70(1), 55-75 (2006)

17. Shattuck, D.W., Leahy, R.M.: Brainsuite: An automated cortical surface identification tool. Medical Image Analysis 8(2), 129-142 (2002)

18. Shi, Y., Tu, Z., Reiss, A., Dutton, R., Lee, A., Galaburda, A., Dinov, I., Thompson, P., Toga, A.: Joint sulcal detection on cortical surfaces with graphical models and boosted priors. IEEE Transactions on Medical Imaging 28(3), 361-373 (2009)

19. Srivastava, A., Klassen, E., Jo, S.H., Jermyn, I.H.: Shape analysis of elastic curves in euclidean spaces. IEEE Trans. on PAMI 33(7), 1415-1428 (2011)

20. Tagare, H.D., O'shea, D., Groisser, D.: Non-rigid shape comparison of plane curves in images. J. Math. Imaging Vis. 16(1), 57-68 (2002)

21. Xu, C., Liu, J., Tang, X.: 2D Shape Matching by Contour Flexibility. IEEE Trans. on PAMI 31(1), 180-186 (2009) 